\title{
CHARACTERISATION OF SMALL-SCALE ATMOSPHERIC WIND- FIELD STRUCTURES USING COHERENT WIND LIDAR WITH SHORT PULSES
}

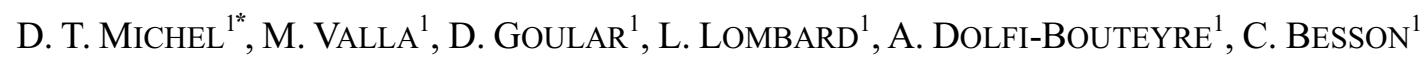

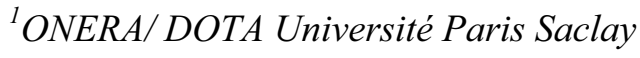 \\ F 91123 Palaiseau - France \\ *Email: david-tomline.michel@onera.fr
}

\begin{abstract}
A lidar design has been developed at ONERA that uses short square pulses ( $75 \mathrm{~ns}$ ) to have a small spatial resolution $(22.5 \mathrm{~m})$ and be able to measure small-scale atmospheric wind-field structures. Results show that the system is able to resolve the small-scale structures of vortices and to measure wind field structures of a turbulent wind field down to $\sim 20 \mathrm{~m}$.
\end{abstract}

\section{INTRODUCTION}

Measuring meter-scale wind field structures are important for a wide variety of physics: in meteorology, to measure atmospheric turbulence [1-6], its effect on ocean elevation [6] and diffusion of pollutants in cities [7], in airport to detect wind shear [8], vortices and measure their time of life [9-11], for airship which are very sensitive to small-scale structures [12], for wind turbine which can be optimized according to the wind structures [13], to perform formation flight [14], or for drone transport. These measurements are generally done remotely using a Coherent Doppler light detection and ranging (lidar) sensor in the Mie regime. This instrument uses a laser to measure the speed of the particles (which is similar to the wind speed) projected along its axis. The backscattered signal is frequency shifted due to particle speed (Doppler Effect) that is determined using heterodyne detection. Improving the spatial resolution of the lidar will improve the restitution of the wind field spatial structure.

To perform this measurement, a first kind of lidar uses pulsed laser $[1,10,11]$ where the length of the pulses $(\Delta \mathrm{T})$ determines the spatial resolution $(\Delta \mathrm{R}=\mathrm{c} \Delta \mathrm{T}$ where $\mathrm{c}$ is the speed of light). If the pulses are long, the spectral resolution, given by the Fourier transform of the pulse (and so the accuracy in velocity measurement), is high but the spatial resolution is low. Up to now, pulses of $400 \mathrm{~ns}-800 \mathrm{~ns}[1,10]$ down to $150-250$ ns $[11,15]$ have been used which limit the resolution to $\sim 50 \mathrm{~m}$. In addition, pulsed laser have a delay before any measurement can be done because of the reflection of the laser on the optics of the system that mixes with the backscattered signal. This blind zone corresponds to a distance of $c \Delta T / 2$ from the output optic. So the longer the pulse, the longer the distance of the first detectable point is.

Another technique consists in using a lidar with continuous wave laser $[11,16]$ that is focused at the region of interest. In this case, the spatial resolution is given by the Rayleigh length of the system over which the backscattered signal is much larger than the signal coming from the other regions. However, such lidar only allows performing the measurement at one location each time which makes the measurement of wind field structures more difficult. In addition, it requires a large output optic to obtain a small Rayleigh length and minimize the spatial resolution. Such optics are expensive and less practical in particular for airborne applications. Furthermore, it is sensitive to large reflections (due to solid target or clouds behind the focus region) that produce a backscattered signal that mixes with the useful signal and corrupts it.

In this article, a pulsed lidar that has been set up at the ONERA is presented. It emits short pulses $(\sim 75 \mathrm{~ns})$ to enable measuring meter-scale wind field structures. The pulse length results in a spatial resolution of 22.5 $\mathrm{m}$ and limits the blind distance to $15 \mathrm{~m}$ ahead of the exit lens.

\section{DESIGN OF THE LIDAR}

The lidar was designed to measure the wind field velocity of wake vortices perpendicular to the path of a plane [Fig. 1]. A short pulse lidar was chosen because the amount of reflecting particles was large and the vortices induced short wind field structures.

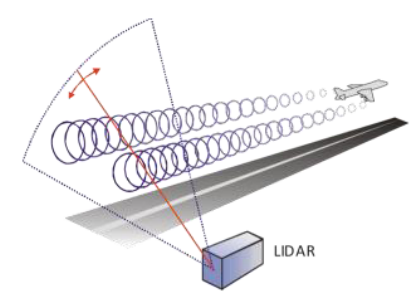

Figure 1: Schematic of the wake vortex characterization by the lidar. 


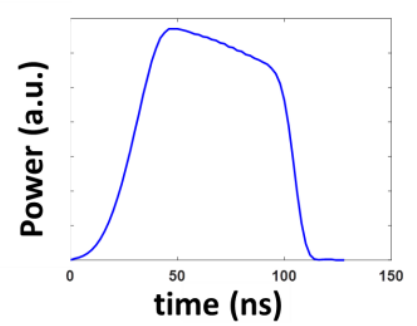

Figure 2: Measured lidar pulse time profile.

The lidar uses a master oscillator power fiber amplifier configuration that emit at 1545-nm [17]. It delivers short pulses with narrow linewidth (close to transform limited) appropriate for heterodyne detection. Their temporal profiles -so called "square" pulse- were shaped with sharp edges (see Fig. 2) to minimize the blind zone, optimize the spatial resolution and maximize the energy per pulse. The pulse shape has intensity at FWHM of $75 \mathrm{~ns}(\sim 100 \mathrm{~ns}$ at $-30 \mathrm{~dB})$ which results in a spatial resolution of $22.5 \mathrm{~m}$ and a blind distance of $15 \mathrm{~m}$ [that correspond to the strong signal at the left of Fig. 2 ].

\section{SIGNAL PROCESSING ON VORTEX}

The lidar has been used on the field to collect experimental data in order to localize vortex center and characterize the induced wind field
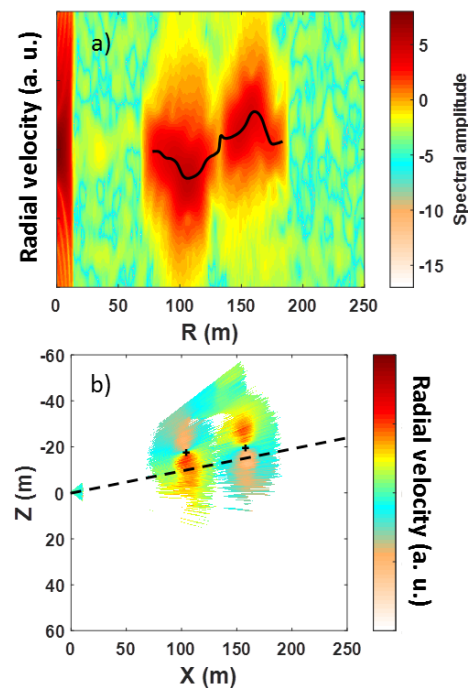

Figure 3: a) frequency versus time plot: Comparison of the lidar spectra measured at $\theta=-5.5^{\circ}$ [dashed line in $b)]$ as a function of distance ( $R$ ). The wind velocity corresponds to the maximum spectral amplitude (black curve). In the figure, the frequencies are converted into velocity. b) Wind field velocities measured during a lidar scan.
Figure 3 a) shows the spectra of the lidar heterodyne current, calculated at each time bin. In the figure, the time bins are converted into distance $(\mathrm{R}=\mathrm{ct} / 2$ where $\mathrm{t}$ is the time bin) and the frequencies are converted into velocity $\left[\mathrm{V}=-\lambda / 2\left(\mathrm{f}-\mathrm{f}_{\mathrm{IF}}\right)\right.$ where $\lambda$ is the laser wavelength, $f$ is the frequency and $f_{I F}$ is the frequency shift of the pulses]. For each bin, the velocity is obtained by determining the maximum of the spectrum.

The wind field velocity is measured using a scan of the lidar between $-20^{\circ}$ and $20^{\circ}$. Figure 2 b) shows an example of modelled wind field measurement for a vortex structure.
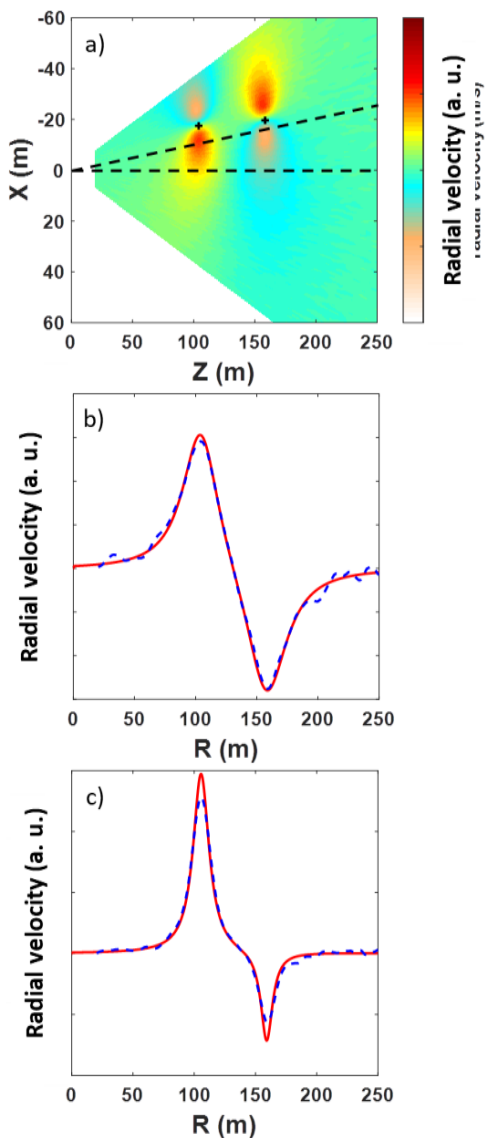

Figure 4: a) Simulated lidar wind field measurement for a two-vortex wind field structure. Comparison of the simulated wind velocity (red curves) and the wind velocity determined from the maximum of the synthetic lidar spectra (dashed blue curves) at an angle $\theta=0^{\circ}$ (b) and $\theta=-5.5^{\circ}(c)$.

The spatial resolution for wake vortex measurement was estimated using simulations. A vortex wind field was determined using the Hallock-Burham model [18] that best fit the data shown in Fig. 3 b). The lidar was simulated to determine the spectra for angles between $20^{\circ}$ and $20^{\circ}$. Figure 4 a) shows the retrieved wind field obtained from the maximum of the synthetic spectra. 
The vortex wind field structures are clearly observables with the positive and negative wind around the vortex core. Experimentally this structure is also well shown which allows determining precisely the vortex core. Post-treatments show that the core position can be determined within less than $1 \mathrm{~m}$.

Figure $4 \mathrm{~b}$ ) and $4 \mathrm{c}$ ) shows the effect of the lidar on the wind profile. At $\theta=0^{\circ}$, the lidar axis is at $\sim 15 \mathrm{~m}$ from the first vortex core position and $20 \mathrm{~m}$ from the second vortex core position. In this case, the lidar allows measuring precisely the velocity profile. In the second case $\left(\theta=-5.5^{\circ}\right)$, the lidar axis is at $5 \mathrm{~m}$ (respectively 3 $\mathrm{m}$ ) from the first (respectively second) vortex. In this case, the maximum velocity is under estimated by $8 \%$ and $10 \%$ respectively. In conclusion, the lidar allows measuring vortex wind velocities with an excellent precision affecting only the maximum velocity between $3 \mathrm{~m}$ and $8 \mathrm{~m}$ from the vortex core with a maximum difference of $10 \%$.

\section{SIGNAL PROCESSING ON TURBULENT WIND FIELD}

A second application of the lidar was tested to characterize turbulent wind field.
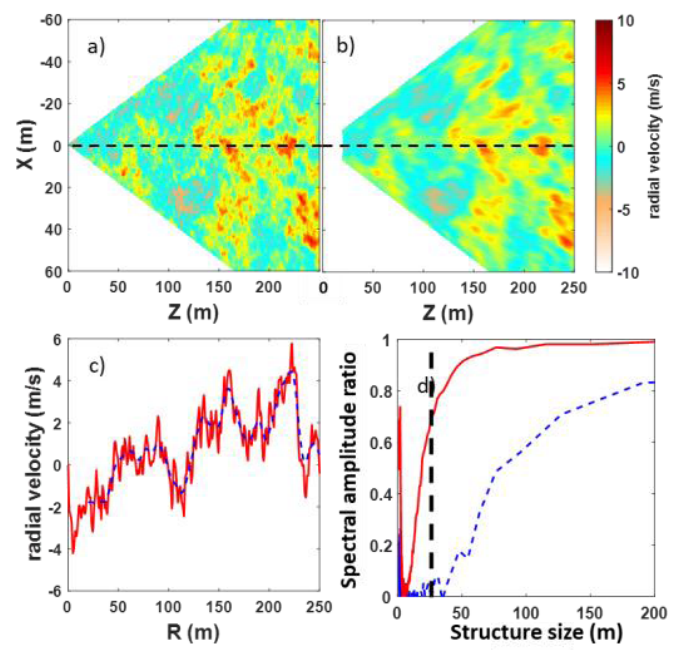

Figure 5: a) Simulated turbulent wind field for a moderate turbulence. b) Velocities determined after processing of the simulated lidar spectra using the wind field in a). c) Comparison of the simulated wind profile at $\theta=0^{\circ}$ with the wind profile determined from the synthetic lidar spectra. Comparison of the spectral amplitude of the velocity profiles as a function of the size of the velocity structure for the 75-ns square pulse (solid red curve) and for an energy equivalent 200-nsFWHM Gaussian pulse (dashed blue curve). For the 75-ns pulse lidar, the spatial resolution of the lidar of about $25 \mathrm{~m}$ is plotted (dashed black line).
Figure 5 a) shows a turbulent wind field simulated using a Von Karman model $[19,20]$ with a characteristic scale length of $200 \mathrm{~m}$ and a velocity variance of $3.2 \mathrm{~m} / \mathrm{s}$ (typical parameters for moderate atmospheric turbulence). The lidar was simulated to determine the spectra.

In Fig. $5 \mathrm{~b}$ ), we see that after processing of the synthetic lidar spectra, most of the wind structure can be distinguished. This is more apparent when the velocity profiles obtained for one line of sight are compared [Fig. $5 \mathrm{c}$ )]. It shows that the amplitude of the high spatial frequencies of the velocity has a low magnitude (according to the model) so that the error in velocity is lower than $1 \mathrm{~m} / \mathrm{s}$ throughout the profile.

To estimate more accurately the lidar filtering on the amplitude of the spatial frequencies of the velocity, we have calculated the Fourier transform of the velocity profiles for each angle for the simulated wind field and for the reconstructed wind field. The spectral amplitude ratio was obtained by dividing the spectral amplitudes of the reconstructed profiles by ones of the simulated profiles [Fig. 5 d)]. This ratio was determined using a $\chi^{2}$ analysis over all the lineouts. It was plotted as a function of the structure sizes that correspond to the inverse of the spatial frequency. Figure 4 d) shows that the amplitude ratio remains large until the size of the structures gets lower than the spatial resolution of the lidar $(22.5 \mathrm{~m})$. In addition, using this curve, the statistic of measured frequencies can be reconstructed up to $\sim 10$ $\mathrm{m}$ by dividing the measured amplitude by this factor.

In Fig. 5 d), this curve was compared with the curve that corresponds to the reduction of the spectral amplitude ratio induced by a lidar that uses a 200-ns Gaussian pulse shape with equivalent energy per pulse. It shows that, for the 200-ns pulse shape, this ratio gets very small $(<0.1)$ below $60 \mathrm{~m}-70 \mathrm{~m}$ (which roughly corresponds to the spatial resolution for this lidar) and is significantly lower than for the 75-ns pulse shape for all the structure sizes shown here. This shows that this new pulse shape design results in a strong improvement of the ability of the lidar to measure small scale structures.

\section{CONCLUSION}

We have designed and tested a special fiber lidar to retrieve wind field velocity with a high spatial resolution. It has been tested experimentally on wake vortices. Its performances on turbulent wind field have been modelled and a strong improvement compared to 200-ns Gaussian pulse is expected.

\section{ACKNOWLEDGEMENTS}

The authors wish to acknowledge Airbus for the support of this study. 


\section{REFERENCES}

[1] W. L. Eberhard and R. E. Cupp J. Atmos. Oceanic Technol 6 809-819 (1989)

[2] R. Frehlich J. Atmos. Oceanic Technol 14 54-75 (1997)

[3] R. Frehlich and R. Cornman Appl. Opt. 38 74567466 (1999)

[4] R. Frehlich and R. Cornman J. Atmos. Oceanic Technol 19 355-366 (2006)

[5] S. Florian et al. J. Phys.: Conf. Ser. 318072019 (2011)

[6] M. Shinozuka and C. M. Jan J. of Sound and Vibration 25(1) 111-128 (1972)

[7] R. Frehlich et al. J. of appli. Meteo. And clim. 45 821-837 (2006)

[8] R. Targ, et al. Appl. Opt., 30 2013-2026 (1991)

[9] M. Harris et al. Aerospace Sci. and Tech. 6 325331 (2002)

[10] F. Köpp et al. J. Atmos. Oceanic Technol 21 194206 (2004)

[11] A. Dolfi-Bouteyre et al. AerospaceLab Journal AL01-07 (2009)

[12] K. El Omari et al., Monografías del Seminario Matemático García de Galdeano 31, 545-554 (2004)

[13] T. Mikkelsen et al. Proceedings online European Wind Energy Association (2010)

[14] S. Ning, A., Flanzer, T. C., \& Kroo, I. M. (2011). Aerodynamic performance of extended formation flight. Journal of aircraft, 48(3), 855-865

[15] S. W. Henderson et al. Opt. Lett., 16, 773-775 (1991)

[16] J.-P. Cariou et al. 13th Coherent Laser Radar conference (CLRC), Kamakura (2005).

[17] A. Dofli-Bouteyre et al. 19th Coherent Laser Radar conference (CLRC), Okinawa (2018).

[18] D. C. Burnham and J. N. Hallock Chicago monostatic acoustic vortex sensor system. Report No. DOT-TSC-FAA-79-103.IV 206pp (1982)

[19] V. Karman Proc. Nat. Acad. Sci. 34 530-539

[20] D. K. Wilson U.S. Army Research Laboratory, ARL-TR-1677 (1998) 